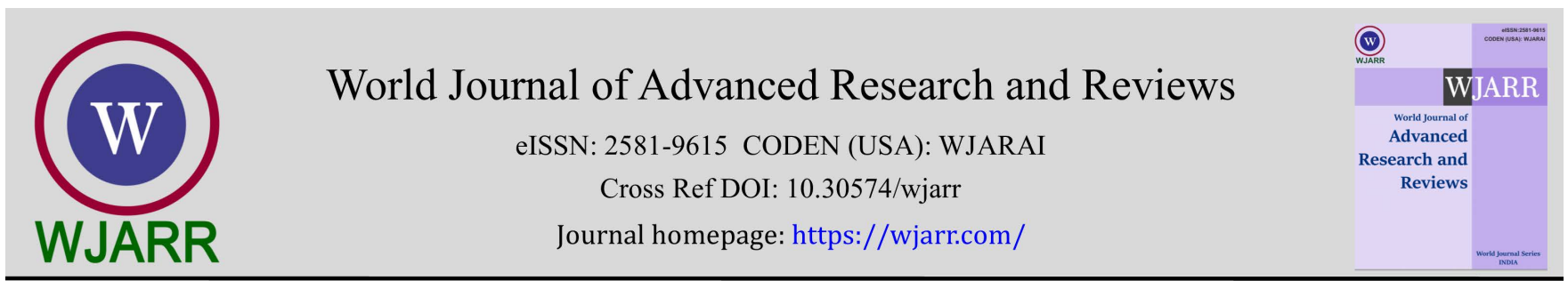

(RESEARCH ARTiClE)

\title{
Assessment of decrease in renal function associated with hypothyroidism
}

\author{
Ayesha Ammar *, Kahkashan Bashir Mir, Sadaf Batool, Noreen Marwat, Maryam Saeed, Adnan Saeed and \\ Shazia Fatima
}

Nuclear Medicine Department, Atomic Energy Cancer Hospital, AECH, NORI, Islamabad, Pakistan.

World Journal of Advanced Research and Reviews, 2021, 12(01), 275-286

Publication history: Received on 22 August 2021; revised on 10 September 2021; accepted on 12 September 2021

Article DOI: https://doi.org/10.30574/wjarr.2021.12.1.0477

\begin{abstract}
Objective: Study was aimed to see the effects of hypothyroidism on GFR as a renal function.

Material and methods: Total of Fifty-eight patients were included in the study. Out of those forty-eight patients were female and the rest were male. Out of fifty eight patients, fifty three patients were of thyroid cancer in which hypothyroidism was due to discontinuation of thyroxine before the administration of radioactive iodine for Differentiated thyroid cancer.Moreover, remaining five patients were post radioactive iodine treatment (for hyperthyroidism) hypothyroid. All of the patients were above eighteen years of age with TSH value $>30 \mu \mathrm{IU} / \mathrm{ml}$. Pregnant and lactating females were excluded.Renal function tests (urea/creatinine, creatinine clearance) and serum electrolytes followed by Tc-99m-DTPA renal scan for GFR assessment (GATES' method) were carried out in all subjects twice during the study, One study during hypothyroid state (TSH $>30 \mu \mathrm{IU} / \mathrm{ml}$ ) and other during euthyroid state (TSH between 0.4 to $4 \mu \mathrm{IU} / \mathrm{ml}$ ). The results of Student's t-test showed significant difference in renal functions (Urea, creatinine, creatinine clearance, GFR values) in euthyroid state and hypothyroid state ( $p$-value $<0.05)$. RESULTS: In case of creatinine the paired t test reveal the mean $1.014 \pm 0.428$, with standard error of 0.669 within $95 \%$ confidence interval, for creatinine clearance $80.11 \pm 14.12$ with standard error of 1.94 within $95 \%$ confidence intervals, for urea the mean $28 \pm 12.13$ with standard error of 1.607 within $95 \%$ confidence intervals and for GFR for individual kidney is $38.056 \pm 8.56$ with standard error of 1.3717 within $95 \%$ confidence interval. There was no difference in the outcome of the 2 groups.
\end{abstract}

Conclusion: Hypothyroidism impairs renal function to a significant level and hence needs to be prevented and corrected as early as possible.

Keywords: Thyroid dysfunction; Glomerular Filtration Rate; Cancer thyroid; Hyperthyroidism

\section{Introduction}

Thyroid gland is a master gland; thus, it regulates majority of the human body's physiological functions. Thyroid gland exerts its effect through thyroid hormones (T3 and T4) on metabolism, body growth, directly affects cardiac myocytes by regulating genes important for myocardial contraction, protein synthesis, and the regulation of many other important hormones [1,2]. If a dysfunction occurs in the thyroid gland leading to the defect in the production of thyroid hormones it can cause various pathologies throughout the body. Thyroid hormones have pre-renal and intrinsic renal effects by which they increase the renal blood flow and the Glomerular filtration rate (GFR) [1].

Thyroid hormones affect both renal morphology and function. They are required for kidney growth and development, and thyroid deficiency results in decreased renal plasma flow and glomerular filtration rate and in impaired urinary

\footnotetext{
${ }^{*}$ Corresponding author: Ayesha Ammar

Nuclear Medicine Department, Atomic Energy Cancer Hospital, AECH, NORI, Islamabad, Pakistan.
}

Copyright (C) 2021 Author(s) retain the copyright of this article. This article is published under the terms of the Creative Commons Attribution Liscense 4.0. 
concentration and dilution [3]. Thyroid hormones also influence membrane transport and electrolyte metabolism, and alterations in mineral metabolism in hyperthyroidism frequently cause calcium nephropathy which affects renal function adversely [4]. Effects of thyroid hormone are 2 layered as there are direct as well as indirect effects. Some are directly affecting the kidney while the indirect actions are a combination of the cardiovascular and systemic hemodynamic effects that influence kidney function. [5]. These direct and indirect effects are illustrated in Fig no 1.

Thyroid hormone directly influences the expression and/or activity of a number of ion channels and transporters (Table 1). Examples of the effects of these changes can be seen clinically in both hyperthyroid and hypothyroid patients. [5].

Table 1Renal tubular ion transporters affected by thyroid hormone

\begin{tabular}{|c|l|c|c|}
\hline Sr.no & transporters & hypothyroidism & hyperthyroidism \\
\hline 1 & Na+-K+ ATPase & $\downarrow$ & $\uparrow$ \\
\hline 2 & H+-K+ ATPase & $\downarrow$ & $\uparrow$ \\
\hline 3 & Na+-HCO3- exchanger & $\downarrow$ & $\uparrow$ \\
\hline 4 & Na+-H exchanger & $\downarrow$ & $\uparrow$ \\
\hline 5 & Na+-Pi IIa exchanger & $\downarrow$ & $\uparrow$ \\
\hline 6 & Na+sulphate exchanger & $\downarrow$ & $\uparrow$ \\
\hline 7 & Na+-K+-2Cl- cotransporter & $\downarrow$ & $\uparrow$ \\
\hline 8 & Na+-Ca2+ exchanger & $\downarrow$ & $\downarrow$ \\
\hline 9 & Cl- channel & $\uparrow$ & $\uparrow$ \\
\hline 10 & AQP 1 and 2 & $\downarrow$ & $\uparrow$ \\
\hline
\end{tabular}

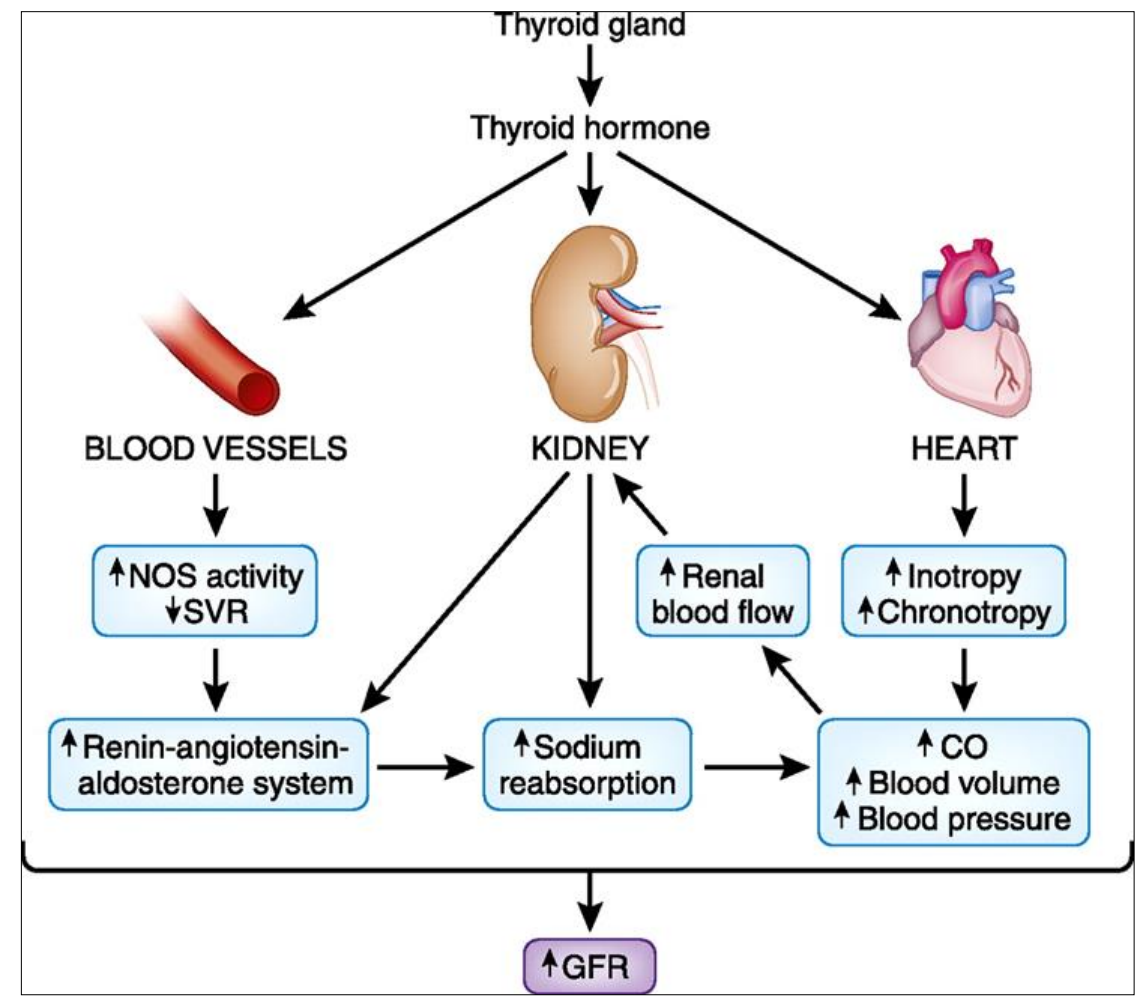

Figure 1 Multiple direct and indirect effects of thyroid hormones on gfr@ copy right from jasn from the renal manifestations of thyroid disease [20]. 
Transporter function is decreased with hypothyroidism and increased with hyperthyroidism or thyroid hormone replacement with the exception of Aquaporin which has the opposite pattern.

Correlation of thyroid hormone levels with CKD has been scarcely explored. Disorders in renal function have been seen to coexist with specific levels of thyroid hormone $[6,19]$. Most of these case reports up till date published rely merely on, using creatinine as an estimation of kidney function. Karanikas and his colleagues performed ${ }^{51} \mathrm{Cr}-\mathrm{EDTA}$ isotopic renal scans in thyroidectomized patients with severe hypothyroidism (mean TSH $70 \pm 23 \mu \mathrm{IU} / \mathrm{ml}$ ) before and after thyroid hormone replacement. A fall in serum creatinine with thyroid hormone replacement $(1.30 \pm 0.44$ versus $1.04 \pm 0.32 \mathrm{mg} / \mathrm{dl}$ ) was associated with an increase in GFR by ${ }^{51} \mathrm{Cr}$-EDTA clearance $(61 \pm 18$ versus $75 \pm 23 \mathrm{ml} / \mathrm{min}$ ) [6]. In another study of hypothyroid patients, estimated renal plasma flow, measured by 131I-hippuran clearance, increased from $542.8 \pm 215.8$ to $717.0 \pm 140.6 \mathrm{ml} / \mathrm{min}$ per $1.73 \mathrm{~m}^{2}$, and GFR, measured with ${ }^{52} \mathrm{Cr}$-EDTA clearance, increased from $99.6 \pm 32.2$ to $125.7 \pm 41.2 \mathrm{ml} / \mathrm{min}$ after thyroid hormone replacement, [7]. thus confirming that changes in levels of serum creatinine in patients with thyroid disorders do reflect actual changes in GFR. Only one case report has been published till date by Sanjay that showed in case of hypothyroidism there is decreased clearance of urea, creatinine and creatinine clearance and thus reduction in GFR and this condition was reverted by the use of levothyroxine. He used $99 \mathrm{mTc}$ DTPA as a renal isotope to measure GFR. [8]. While in another study by Andrew Conner it is suggested that in patients of kidney diseases levels of TSH and FT4 must be taken into account for their treatment. [18]..

\section{Aims and Objectives}

To study the effect of hypothyroidism on renal function using camera based GFR assessment method.

\section{Material and methods}

The study was conducted in Nuclear Medicine department of NORI. It was approved by the ethical review committee of atomic energy cancer hospital NORI. It was a prospective study. Itwas span over a period of 6 months from Jan 2017 till June 2017. Informed consent was taken from the patients. Total of fifty-eight patients were included in the study. Fortyeight patients were female and the rest are male. Out of fifty-eight patients, fifty-three patients were known case of carcinoma of thyroid in whom hypothyroidism was due to discontinuation of thyroxin before the administration of radioactive iodine either for the whole-body scan or for the ablation in the patients of differentiated thyroid carcinoma. Remaining five patients were those who were treated with radioactive iodine for hyperthyroidism.

\subsection{Inclusion Criteria}

All of the patients were above eighteen years of age with TSH value $>30 \mathrm{Iu} / \mathrm{ml}$.

\subsection{Exclusion Criteria}

Pregnant and lactating females and those with the co morbid conditions such as hepatic or renal failure were excluded.

\subsection{Protocol}

Following investigations are carried out in each patient after informed consent

- $\quad$ Baseline renal dynamic study with 99mTc-DTPA with GFR calculation- when the patient is euthyroid

- Renal dynamic study with 99mTc-DTPAscan with GFR calculation- when patient has raised TSH value (>30 $\mathrm{IU} / \mathrm{ml}$ )

- $\quad$ Renal function tests (urea/creatinine) at both occasions

- Serum electrolytes were done base line for all of the patients, if at base line serum electrolytes were low, then after giving T4 and performing scan serum electrolytes were repeated.

- Creatinine clearance in $\mathrm{ml} / \mathrm{min}$ was performed as a baseline study in every patient and only repeated in those cases where there was a decrease in creatinine clearance.

- For every patient ultrasound studies were also performed as a routine base line investigation. 


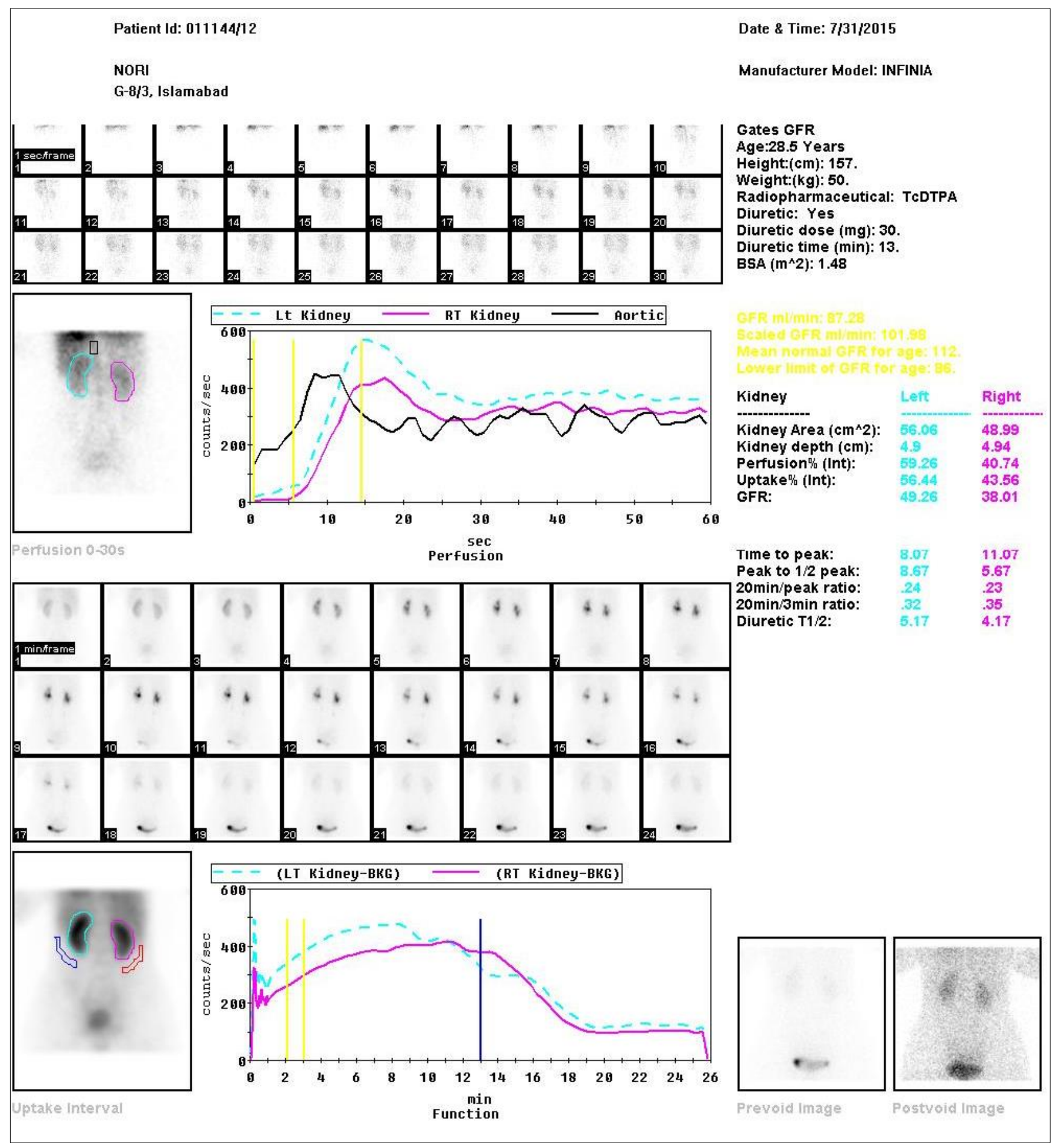

Figure 2 After withdrawal of thyroxine

Imaging was performed on dual headed gamma camera. The processing and quantitative analysis was done using Xeleris model H2400PLJ and description Infinia GP3. ${ }^{99 \mathrm{~m}}$ Tc DTPA according to the weight of the patient was injected under the gamma camera and the dynamic study was performed in posterior mode for 25-30 minutes. Initially the perfusion images were taken for2sec/frame for 1 minute to see the aorta blood flow and its relation to the renal artery flow and then the dynamic study in which $1 \mathrm{frame} /$ minute was taken for radiotracer uptake, corticopelvic radiotracer transit and then excretion. After the acquisition of the study region of interest were drawn on aorta, and on both kidneys. Relative renal uptake, renograms and Glomerular function rates were calculated. The same scan with same acquisition parameters were repeated after the continuation of thyroxine when the patient has attained the euthyroid status. Laboratory testing of TSH, FT4, urea, creatinine and creatinine clearance was performed by a third generation 
ultrasensitive electrochemiluminescence immunoassay (Elecsys on Modular and Cobas e 602 instruments, Roche Diagnostics, Penzberg, Germany).

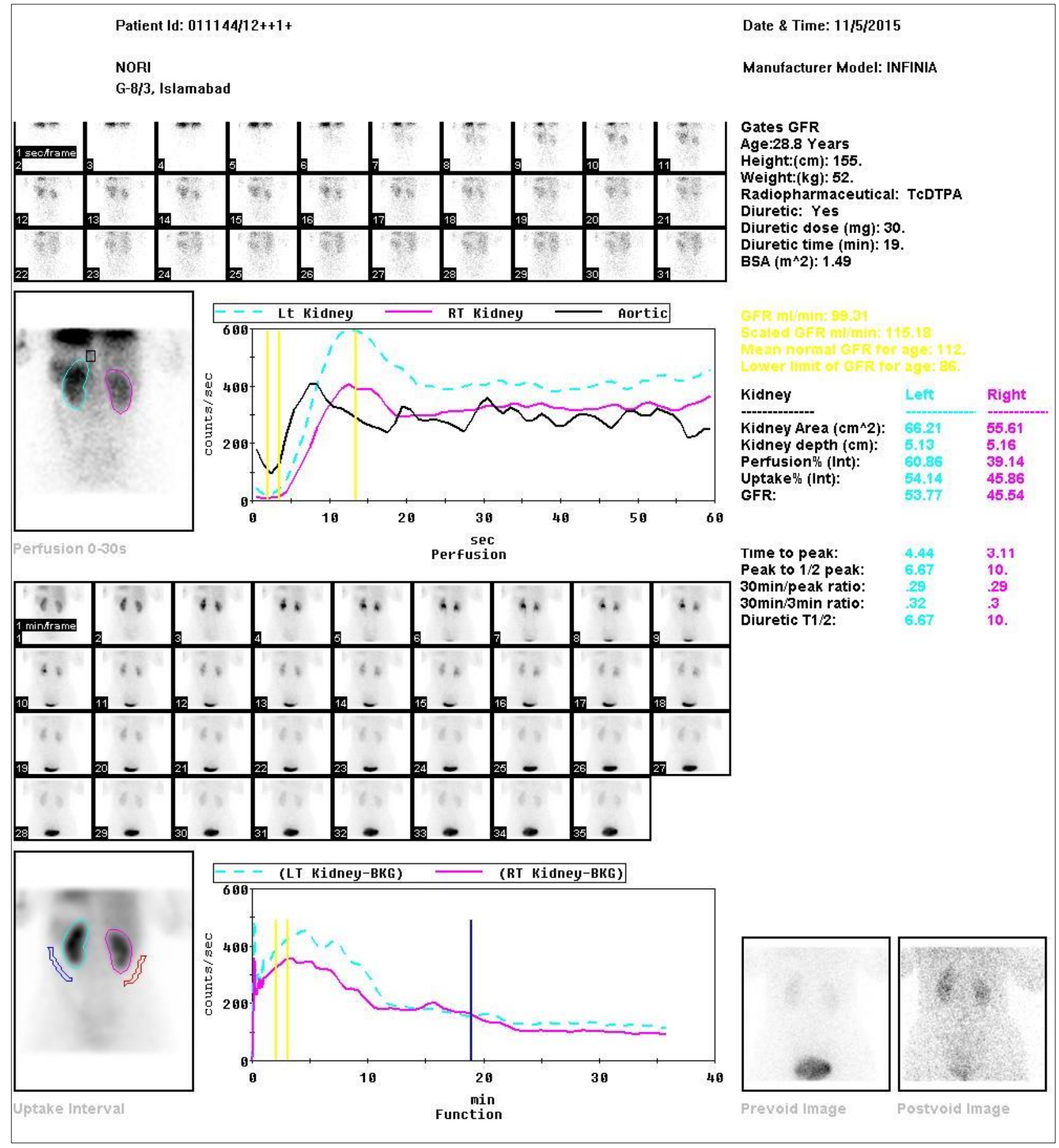

Figure 3 After starting thyroxine GFR of both kidneys in the bilateral good function

The data was then statistically analyzed on Microsoft Excel 2010 and SPSS version 17.The significance of difference in the scan results in between off Thyroxine and on thyroxine was calculated using nonparametric student $\mathrm{T}$ test at cut off value of 0.05 were considered significant. 
World Journal of Advanced Research and Reviews, 2021, 12(01), 275-286

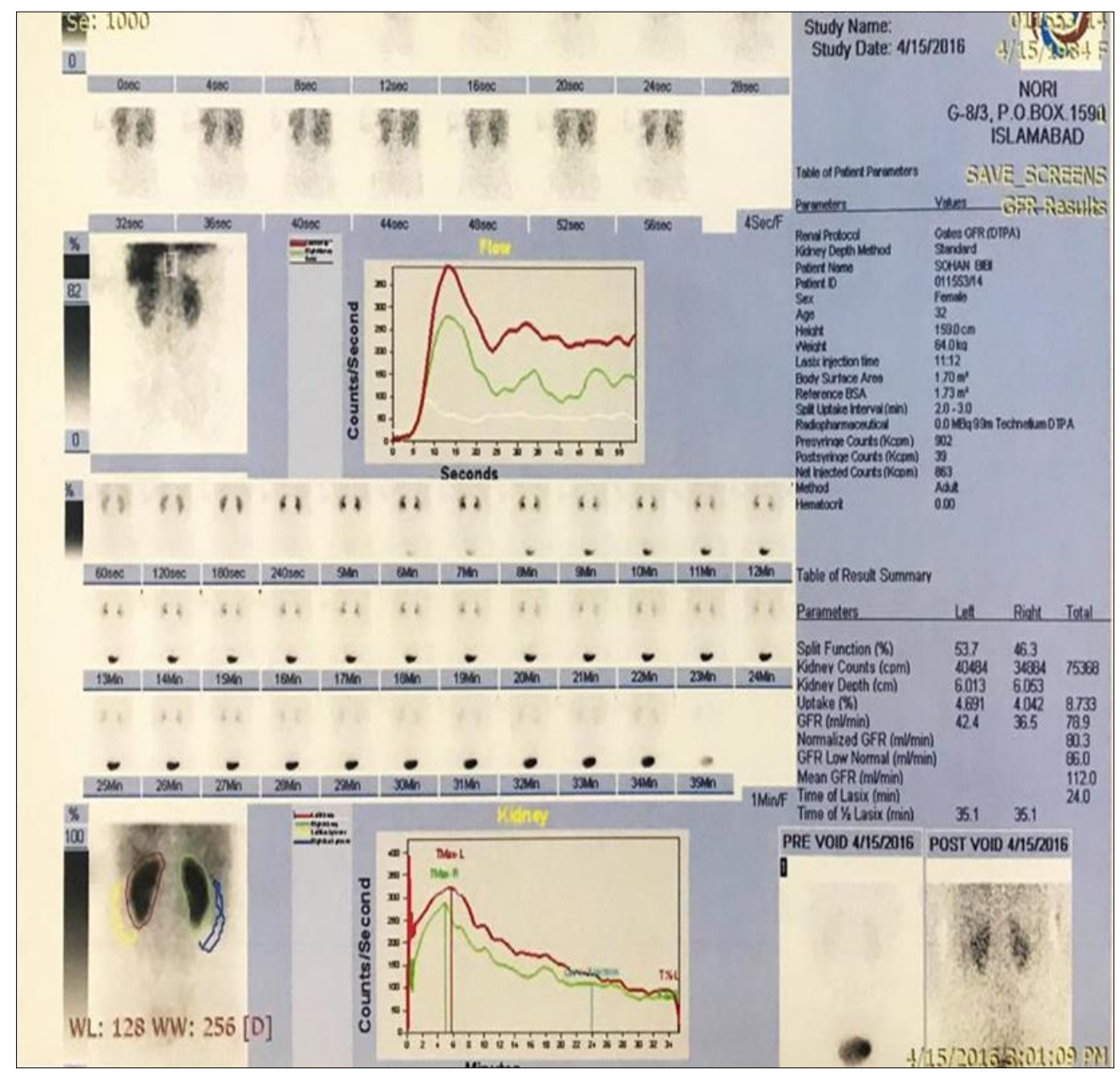

Figure 4 Patient in a state of hypothyroidism with reduction in GFR

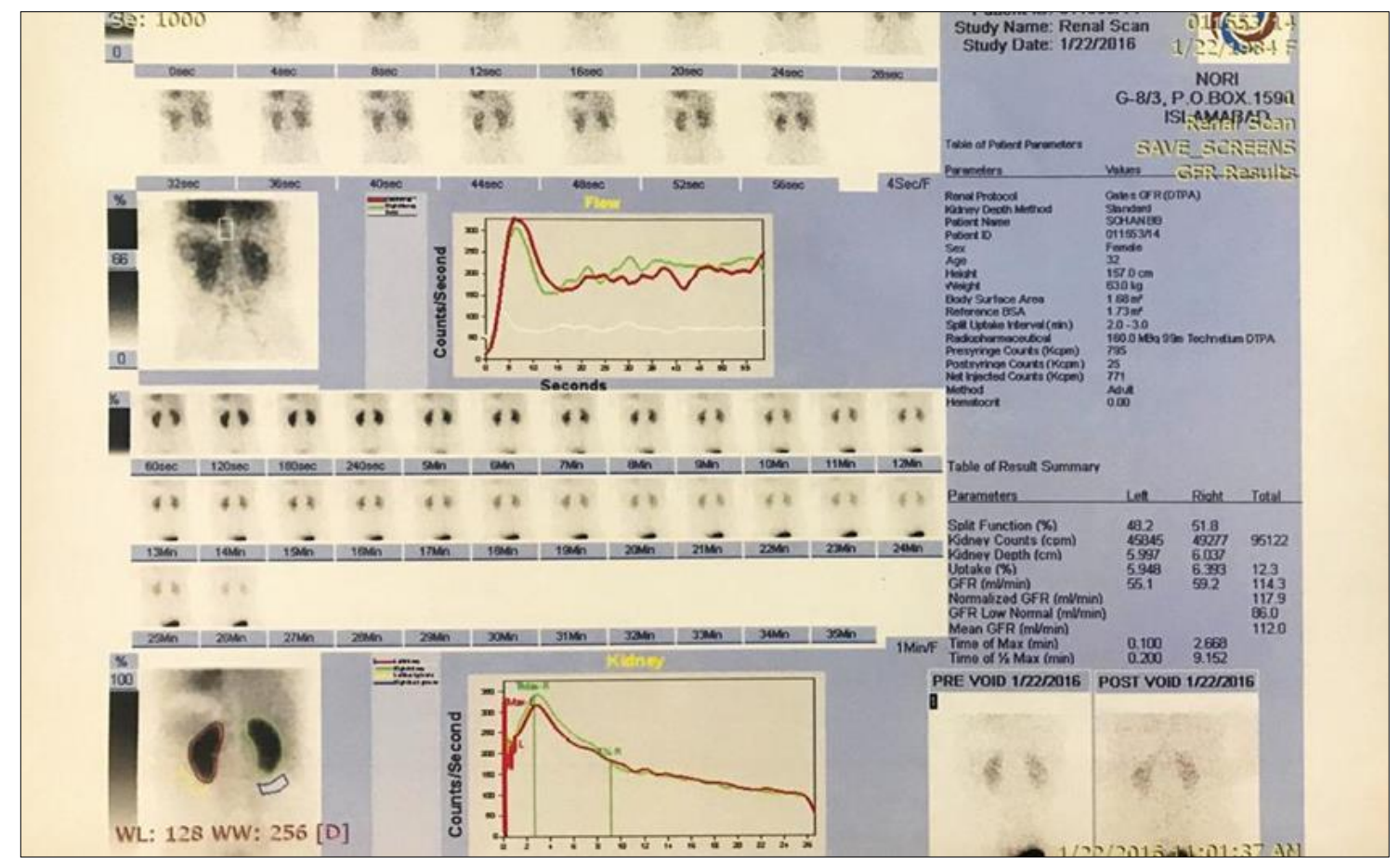

Figure 5 Renal scan performed after attaining the euthyroid status showing the normalization of GFR 


\section{Results}

Table 2 Sr \# Characteristics of Study Population Values

\begin{tabular}{|c|l|c|}
\hline $\mathbf{1}$ & \multicolumn{1}{|c|}{ Total female pts } & $\mathbf{4 8}$ \\
\hline 2 & Total male pts & 10 \\
\hline 3 & Mean age(yrs) & $45.27 \pm 13.9$ \\
\hline 4 & Median height $(\mathrm{cm})$ & $155.22 \pm 8.6$ \\
\hline 5 & Median Weight $(\mathrm{kg})$ & $55.72 \pm 9.1$ \\
\hline
\end{tabular}

The results of Student's t-test showed significant difference in renal functions such as Urea, creatinine, creatinine clearance, GFR values in euthyroid state and hypothyroid state ( $p$-value $<0.05$ ). In case of creatinine the paired t test reveal the mean and S.D of $1.01 \pm 0.42$, with standard error of 0.669 within $95 \%$ confidence interval, for creatinine clearance the mean and S.D were $80.11 \pm 14.12$ with standard error of 1.94 within $95 \%$ confidence intervals, for urea the mean and S.D were $28 \pm 12.13$ with standard error of 1.607 within $95 \%$ confidence intervals and for gfr for individual kidney the mean and S.D were 38.06 \pm 8.56 with standard error of 1.3717 within $95 \%$ confidence interval.

\subsection{Renogram analysis}

Our study also depicts that in addition to the GFR values their is difference in the renal transit times, when given thyroxine there is reduction in time to peak, peak to half peak, 20 minutes to peak and also we can also see that renogram showed good uptake peak followed by satisfactory down slopes. Uptake occurs at 3-5 minutes with an average of $3.8 \pm 0.56$ in the euthyroid state and $7.89 \pm 1.2$ minutes in case of hypothyroidism. As renal function deteriorates, delayed transit of the radiopharmaceutical in the kidney results in an abnormal renogram curve, which can be quantitated by using this index, The 20 minute-to-peak count ratio $(20 \mathrm{~min} /$ maximum count ratio). This is the activity measured in each kidney at 20 minutes and expressed as a percentage of peak curve activity and is often measured for whole kidney and cortical regions of interest. As renal function deteriorates, delayed transit of the radiopharmaceutical in the kidney results in an abnormal renogram curve, which can be quantitied by using this index. In the absence of pelvic calyceal retention, or if only a cortical region of interest is used, a normal 20-minute maximal cortical ratio was averages $0.185 \pm 0.59$ which is in correlation with our study in which we calculated it to be $0.19 \pm 0.73$. While in case of hypothyroidism it can be seen that the uptake peak was after 8 minutes and peak was somewhat blunt and there is a plateau of hold up followed by down slope of excretion as noted in fig no 2 and figure no 4 20-minute maximal cortical ratio was averages $0.38 \pm 0.54$.(17). Figure no 8, 9, 10 showed almost linear correlation of TSH with urea, creatinine and G.F.R.

\subsection{Blood test results}

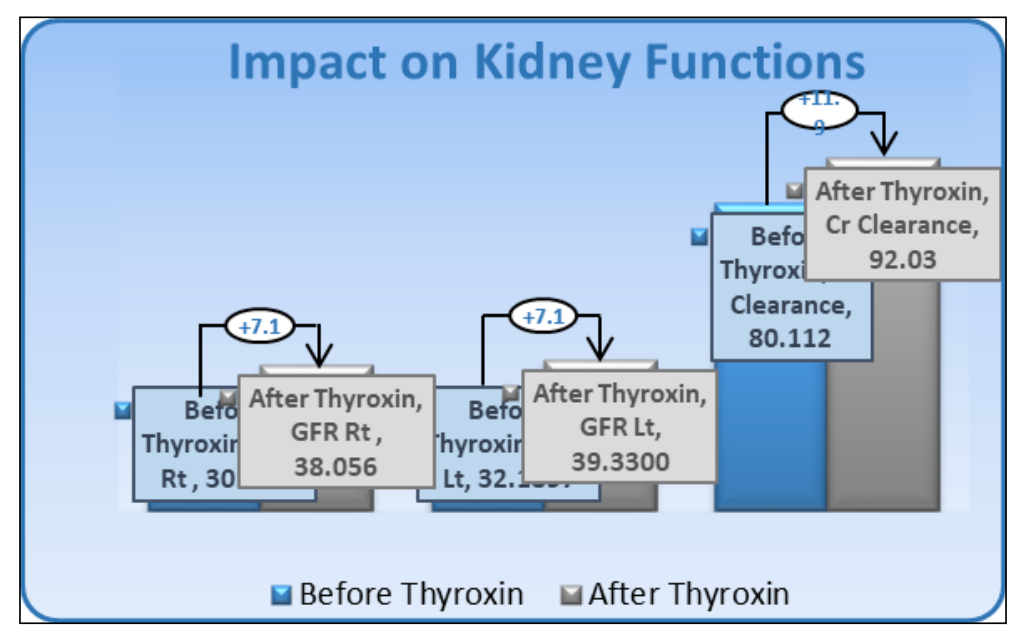

Figure 6 Changes in GFR and creatinine clearance in euthyroid state 


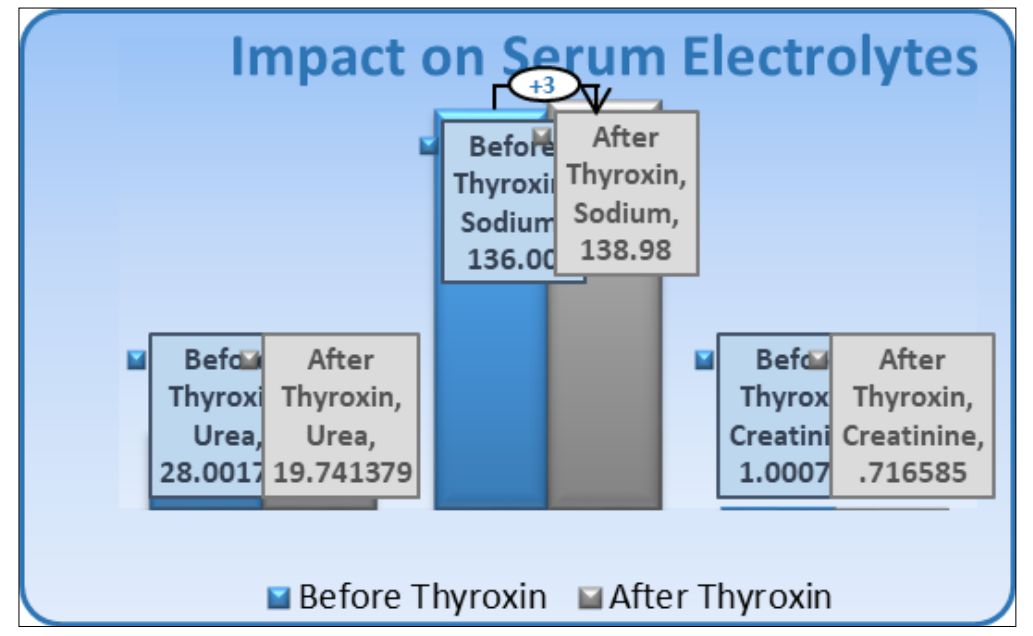

Figure 7 Changes in urea, creatinine and sodium after attaining the euthyroid state

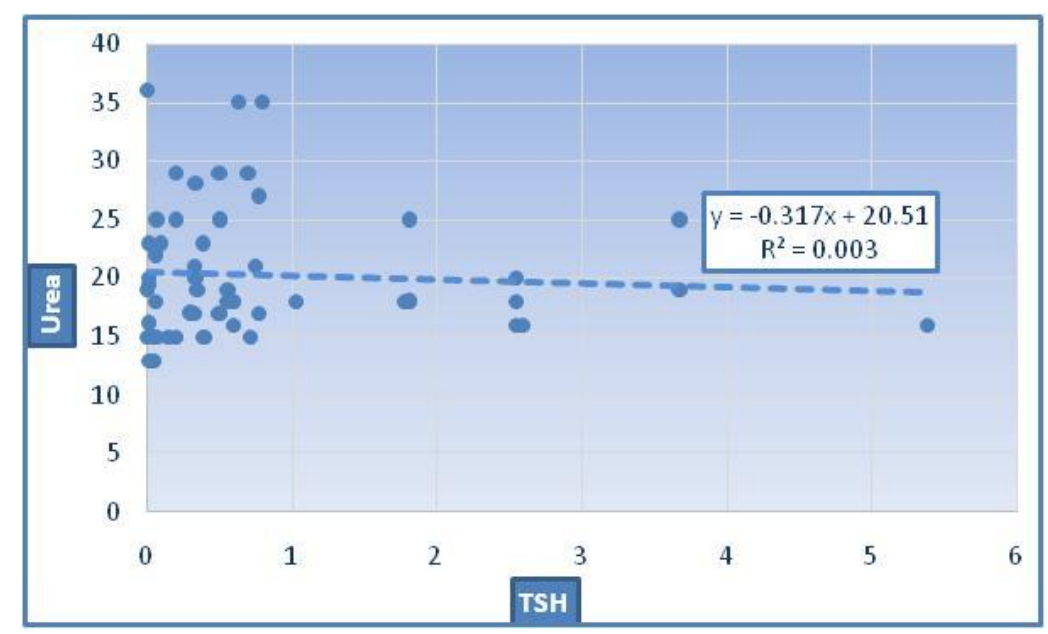

Figure 8 Linear fit curve for urea off Thyroxine showing almost linear correlation between urea and thyroxine.

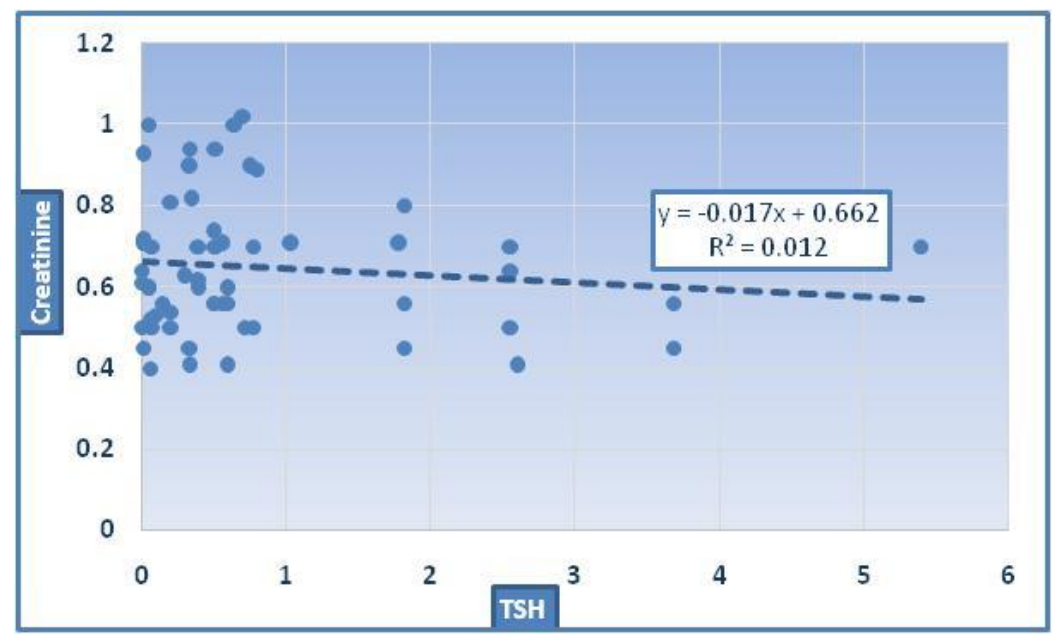

Figure 9 Almost linear correlation of TSH versus creatinine 


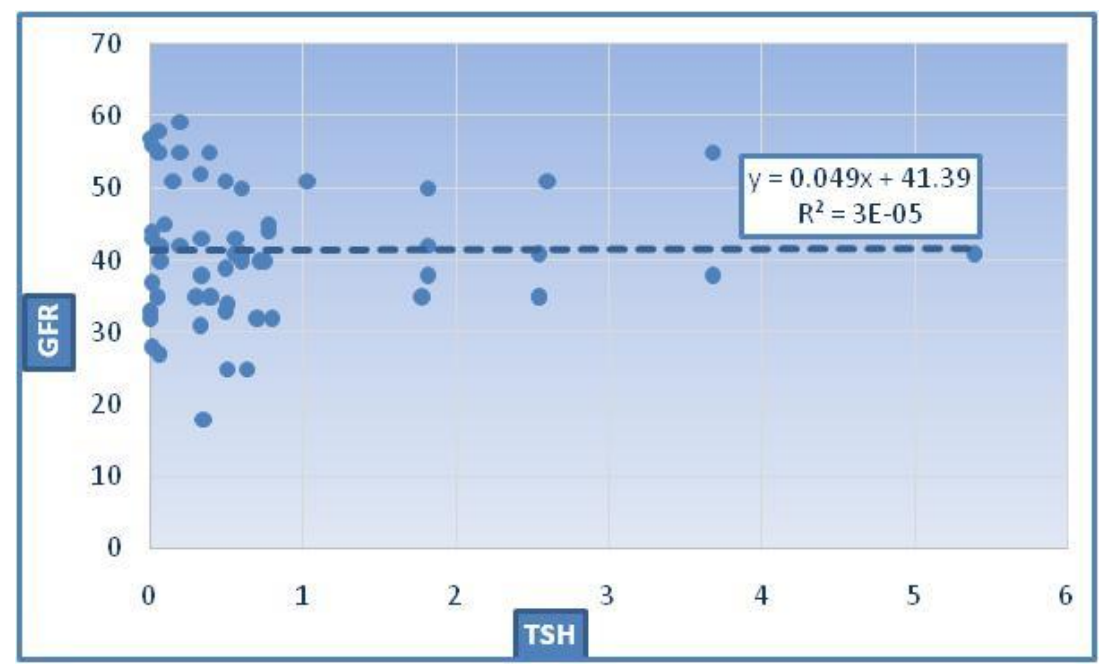

Figure 10 Almost linear correlation of TSH with GFR

Hypothyroidism leads to retention of sodium in blood thus we can see in the blood assays that there raised TSH leads to raised serum urea, creatinine, serum sodium levels thus decreasing the creatinine levels. While these changes reverted to normal after achieving euthyroid status. Creatinine clearance was increased from 80.1 to 92 , also had an impact on creatinine as its value decreased from 1 to 0.7 and to a lesser extent on urea as its value decreased from 30 to 19 and the value of $\mathrm{Na}$ was also restored from 136 to139.

\section{Discussion}

Thyroid hormones mainly T4 and to a lesser extent T3 affects nearly every organ system in the body. It is produced and secreted by the thyroid gland under the control of the anterior pituitary hormone thyroid stimulating hormone (TSH), which is, in turn, regulated by hypothalamus. [3].

Thyroid hormone influences the kidney size, weight, and structure both during development and in adults. Several Histologic studies document the effects of thyroid hormone on cortical and outer medullary tubular segments, particularly involving the proximal tubule, distal convoluted tubule, and medullary thick ascending limb [4-6]. It has been observed in neonatal rats, hypothyroidism is the cause of the decrease in kidney size and weight, tubule length and diameter, and, to a lesser extent, glomerular volume. [9].. These changes invariably reverse with thyroid hormone replacement.

Children with congenital hypothyroidism have reduced renal mass and a higher prevalence of renal and urologic abnormalities, including dysplastic kidney, renal agenesis, ectopic kidney, hydronephrosis, posterior urethral valves, and hypospadias $[10,11]$.

\subsection{GFR and thyroid dysfunction}

In a study conducted on 29 patients by S.H Kriessman he concluded that there is a consistent and reversible elevation of serum creatinine values in the hypothyroid state. [12]. The importance of understanding the impact of thyroid dysfunction on renal function is highlighted by recent studies indicating subclinical and clinical hypothyroidism is common in patients with estimated GFR $<60 \mathrm{ml} / \mathrm{min}$ per $1.73 \mathrm{~m}^{2}$, begging the question of whether hypothyroidism might be contributing to the low GFR in some of these individuals. [12,13]

Elevation of levels of serum creatinine can occur within as little as 2 weeks of significant hypothyroidism. These levels typically normalize rapidly with thyroid hormone replacement after short periods of hypothyroidism [12]. but slower and incomplete recovery has been noted with more prolonged periods of severe hypothyroidism. Similarly, multiple human and animal studies demonstrate a decreased serum creatinine in the setting of hyperthyroidism, which is similarly reversible upon treatment. [13].

One of the electrolyte imbalance caused by the hypothyroidism is hyponatremia and is due to impaired water excretion. Multiple studies in hypothyroid animals demonstrate reduced capacity to achieve maximal urinary dilution due to nonosmotic arginine vasopressin release, as well as impaired urinary concentrating ability, increased urinary sodium 
excretion, increased fractional excretion of sodium, and impaired tolerance of sodium restriction [14, 16]. Experiments have shown that these animals exhibit decreased $\mathrm{Na}+-\mathrm{H}+$ exchanger and $\mathrm{Na}+-\mathrm{Pi}$ co transporter activity. A small study in five hypothyroid men given an acid load demonstrated a decreased ability to acidify the urine. (15) In our present study we also saw that in the absence of thyroxine there is increase in the level of serum creatinine that is restored after the attainment of euthyroid status.

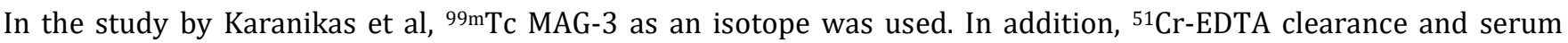
creatinine concentrations were also determined. The serum creatinine concentrations were significantly increased in hypothyroidism as compared with the concentrations after thyroxine substitution $(1.30+/-0.44 \mathrm{vs} .1 .04+/-0.32 \mathrm{mg} / \mathrm{dl}$, $\mathrm{p}<0.05)$. According to them, the glomerular filtration rate was significantly lower in hypothyroidism than after treatment (61+/- 18 vs. $75+/-23 \mathrm{ml} / \mathrm{min})$. In contrast, they did not find any significant change in the renographic parameters for 99mTc-MAG(3) before and after treatment (total excreted activity 20 min after administration $51+/-12$ vs. 54 +/- 14\%; T(max) left:right $4.2+/-1.77: 3.91+/-1.06$ min vs. $4.1+/-1.66: 4.4+/-1.96$ min) [6].In our study we also found that G.F.R of kidney before the starting of thyroxine was $32.067 \mathrm{ml} / \mathrm{min}+/-8.02$ of an individual kidney that was restored to $38.52 \mathrm{ml} / \mathrm{min}+/-8.37$ for an individual kidney.

They did not find any influence of thyroid hormones on the outcome of ${ }^{99 m T c-M A G(3) ~ r e n o g r a p h y . ~ A s ~}{ }^{99 m}$ Tc-MAG(3) reflects the tubular function, it seems that the renal hemodynamic changes in severe hypothyroidism mainly affect the glomerular function. In general, the glomerular filtration rate reduction seems to be reversible after hormone substitution therapy; however, care has to be taken in patients with renal insufficiency.

In the study by Villibona et al , they assessed blood volumes andglomerular filtration rate (GFR) in 17 patients with overt primary hypothyroidism and in 15 of these patients when in euthyroid state after substitutive therapy and the same measurements in eight patients with subclinical hypothyroidism. [7].GFR increased after thyroxine therapy (p < 0.05). In the subclinical group, blood volumes and renal function were similar to those found in the other group of patients when in the euthyroid state.

In another study by YUKI TANAKA they found that e GFR gradually decreased with elevated serum TSH levels and that the significant linear correlation between serum TSH levels and e GFR values. [19]. In our study we also saw that there is almost linear correlation between the TSH value and GFR values.

Increased levels of TSH have a direct effect on (GFR) and have an increased risk of developing chronic kid ney disease. Effective hormone replacement therapy has been shown to postponed the progression to end-stage renal disease in sub-clinically hypothyroid patients with renal insufficiency. In a study conducted by Scharier he concluded that the an increase in TSH between 12 and 24 months after kidney transplantation leads to a significant decrease in eGFR, which strengthens the concept of a kidney-thyroid-axis [21].

In the case report by Sanjay he also showed it scintigraphically that there is reduction in GFR in the absence of thyroxine he also used 99m Tc DTPA revealed a mildly compromised cortical function with adequate clearance of right kidney and adequate cortical function with adequate clearance of left kidney, showing a remarkable recovery of renal function with THRT.

But he did not comment about the values of the transit times. The uptake value is of 3-5 minutes normally. While in case of euthyroid state we found to have uptake values at with an average of $3.8 \pm 0.56$ and $7.89 \pm 1.2$ minutes in case of hypothyroidism. As renal function deteriorates, delayed transit of the radiopharmaceutical in the kidney results in an abnormal renogram curve, which can be quantitated by in the form of 20 minute-to-peak count ratio (20 min/maximum count ratio). This is the activity measured in each kidney at 20 minutes and expressed as a percentage of peak curve activity and is often measured for whole kidney and cortical regions of interest. As renal function deteriorates, delayed transit of the radiopharmaceutical in the kidney results in an abnormal renogram curve, which can be quantitied by using this index. In the absence of pelvic calyceal retention, or if only a cortical region of interest is used, a normal 20 minute maximal cortical ratio was averages $0.185 \pm 0.59$, in our case of euthyroid it was calculated to be about the $0.19 \pm 0.73$. While in case of hypothyroidism it can be seen that the uptake peak was after 8 minutes and peak was somewhat blunt and there is a plateau of hold up followed by down slope of excretion as noted in fig no 2 and figure no 4 20-minute maximal cortical ratio was averages $0.38 \pm 0.54$ [17].

\section{Conclusion}

Hypothyroidism, irrespective of the cause, impairs renal function to a significant level and hence needs to be prevented and corrected as early as possible. 


\section{Compliance with ethical standards}

\section{Acknowledgments}

I like to thank Our Director Dr. MuhammadFaheem, and our department.

\section{Disclosure of conflict of interest}

No conflict of interest is present.

\section{Funding}

No grant/fund was taken for this project.

\section{Statement of ethical approval}

Ethical approval was granted by the Research Training and Monitoring Cell (RTMC) of NORI, in JUNE 2016, RTMC 3/174- 2016, and informed consent was obtained from the patients.

\section{Statement of informed consent}

Informed consent was taken from the patients.

\section{References}

[1] G Basu, A Mohapatra. Interactions between thyroid disorders and kidney disease, Indian Journal of Endocrinology and Metabolism. 2012; 16(2): 204-213.

[2] Klein I, Danzi. Thyroid disease and the heart. Circulation. 2007; 116: 1725-1735.

[3] Brent GA. The molecular basis of thyroid hormone action. N Engl J Med. 1994; 331: 847-853.

[4] Davis RG, Madsen KM, Fregly MJ, Tisher CC. Kidney structure in hypothyroidism. Am J Pathol. 1983; 113: 41-49.

[5] Mariani LH, Berns JS. The renal manifestations of thyroid disease. J Am SocNephrol. 2012; 23: 22-6.

[6] Karanikas G, Schütz M, Szabo M, Becherer A, Wiesner K, Dudczak R, Kletter K Isotopic renal function studies in severe hypothyroidism and after thyroid hormone replacement therapy. Am J Nephrol. 2004; 24: 41-45.

[7] Villabona C, Sahun M, Roca M, Mora J, Gómez N, Gómez JM, Puchal R, Soler J. Blood volumes and renal function in overt and subclinical primary hypothyroidism. Am J Med Sci. 1999; 318: 277-280.

[8] Hypothyroidism presenting as reversible renal impairment: an interesting case report by Sanjay Vikrant, SubhashChander, Satish Kumar \&Dalip Gupta Pages. 2013; 1292-1294.

[9] Salomon MI, Di Scala V, Grishman E, Brener J, Churg J. Renal lesions in hypothyroidism: A study based on kidney biopsies. Metabolism. 1967; 16: 846-852.

[10] Kumar J, Gordillo R, Kaskel FJ, Druschel CM, Woroniecki RP. Increased prevalence of renal and urinary tract anomalies in children with congenital hypothyroidism. J Pediatr. 2009; 154: 263266.

[11] Park SM, Chatterjee VK. Genetics of congenital hypothyroidism. J Med Genet. 2009; 42: 379-389.

[12] Kreisman SH, Hennessey JV. Consistent reversible elevations of serum creatinine levels in severe hypothyroidism. Arch Intern Med. 1999; 159: 79-82.

[13] den Hollander JG, Wulkan RW, Mantel MJ, Berghout A. Correlation between severity of thyroid dysfunction and renal function. ClinEndocrinol (Oxf). 2005; 62: 423-427.

[14] Schmitt R, Klussmann E, Kahl T, Ellison DH, Bachmann S. Renal expression of sodium transporters and aquaporin2 in hypothyroid rats. Am J Physiol Renal Physiol. 2003; 284: 2003.

[15] Oster JR, Michael UF, Perez GO, Sonneborn RE, Vaamonde CA. Renal acidification in hypothyroid man. ClinNephrol. 1976; 6: 398-403.

[16] Åsvold BO, Bjøro T, Vatten LJ. Association of thyroid function with estimated glomerular filtration rate in a population-based study: the HUNT study. Eur J Endocrinol. 2011; 164: 101-5. 
[17] Renal impairment resulting from hypothyroidism by Andrew Connor and Joanne E. Taylor NTD plus. 2008; 1(6): 440-441.

[18] Essentials of Nuclear Medicine and molecular imaging (seventh edition). 2019.

[19] Correlation between Thyroid Stimulating Hormone and Renal Function in Euthyroid Residents of Japan: Results from the Kyushu and Okinawa Population Study (KOPS)

[20] The Renal Manifestations of Thyroid Disease Laura H. Mariani and Jeffrey S. Berns JASN. January 2012; 23(1): $22-26$.

[21] Schairer B, Jungreithmayr V, Schuster M, et al. Effect of Thyroid Hormones on Kidney Function in Patients after Kidney Transplantation. Sci Rep. 2020; 10: 2156. 